\title{
ON PROPERLY SEPARABLE QUOTIENTS OF STRICT (LF) SPACES
}

\author{
W. J. ROBERTSON
}

(Received 28 March 1988)

Communicated by S. Yamamuro

\begin{abstract}
All known Banach spaces have an infinite-dimensional separable quotient and so do all nonnormable Fréchet spaces, although the general question for Banach spaces is still open. A properly separable topological vector space is defined, in such a way that separable and properly separable are equivalent for an infinite-dimensional complete metrisable space. The main result of this paper is that the strict inductive limit of a sequence of non-normable Fréchet spaces has a properly separable quotient.
\end{abstract}

1980 Mathematics subject classification (Amer. Math. Soc.) (1985 Revision): 46 A 05, 46 A 07, 46 A 12.

\section{Introduction}

The problem of the existence of an infinite-dimensional separable quotient of a Banach space has been much studied: see, for example, H. E. Lacey [2] and S. A. Saxon and A. Wilansky [5]. A result of G. Köthe [1, page 431] includes a proof that any non-normable Fréchet space has a quotient isomorphic to $\omega$, the product of a countable number of copies of the scalar field, and so separable.

Clearly an infinite-dimensional topological vector space is separable if and only if it has a dense vector subspace of countable dimension. Here, dimension means the cardinal of a Hamel basis; for convenience, countable is taken to mean infinite and countable.

(C) 1989 Australian Mathematical Society 0263-6115/89 \$A2.00+0.00 
We call a topological vector space properly separable if and only if it has a proper dense vector subspace of countable dimension. Since a complete metrisable topological vector space cannot have countable dimension, it is properly separable if and only if it is separable. In fact this is also true, for the same reason, of a metrisable barrelled locally convex space. For the only barrelled space of countable dimension is the space $\varphi$, the topological direct sum of a countable number of copies of the scalar field, which is not metrisable.

Properly separable quotients of barrelled spaces are discussed in [3] by W. J. Robertson and P. P. Narayanaswami, in connection with the main result of [5].

The notation and terminology are, for the most part, those of [4]. We take quotient to imply quotient by a closed vector subspace.

\section{The main theorem}

LEMMA 1. Suppose that $E$ is a metrisable locally convex space, that $F$ is a closed vector subspace of $E$, and that $\left\{V_{n}: n=0,1,2, \ldots\right\}$ is a decreasing base of absolutely convex neighbourhoods of $o$ in $F$. Then there is a decreasing base $\left\{U_{n}: n=0,1,2, \ldots\right\}$ of absolutely convex neighbourhoods of $o$ in $E$ with $U_{n} \cap F=V_{n}$ for all $n$.

Proof. If $\left\{W_{n}: n=0,1,2, \ldots\right\}$ is any base of absolutely convex neighbourhoods of $o$ in $E$ and if, for each $n, X_{n}$ is an absolutely convex neighbourhood of $o$ in $E$ with $X_{n} \cap F=V_{n}$, then taking $U_{n}$ to be the absolutely convex envelope of $V_{n} \cup\left(X_{n} \cap W_{n}\right)$ will suffice.

COROLLARY. With the notation of the lemma, suppose that, for each $n \geq 1$, $f_{n}$ is a continuous linear form on $F$ with $f_{n} \in V_{n}^{0}$ but $f_{n} \notin \operatorname{span} V_{n-1}^{0}$. Then there is an extension $\bar{f}_{n}$ of $f_{n}$ to a continuous linear form on $E$, with $\bar{f}_{n} \in U_{n}^{0}$ but $\bar{f}_{n} \notin \operatorname{span} U_{n-1}^{0}$.

Proof. If $p_{n}$ is the gauge of $U_{n}$, then $\left|f_{n}(x)\right| \leq p_{n}(x)$ on $F$. The HahnBanach theorem then ensures the existence of an extension $\bar{f}_{n}$ with the required properties.

The next lemma is more or less contained in the result of Köthe mentioned before. We give a brief proof of it in the following formulation, convenient for our purpose. 
Lemma 2. Suppose that $E$ is a non-normable Fréchet space. Then

(a) there is a base $\left\{U_{n}: n=0,1,2, \ldots\right\}$ of absolutely convex neighbourhoods of o with $U_{n}^{0} \nsubseteq \operatorname{span} U_{n-1}^{0}$ for $n=1,2, \ldots$;

(b) if $\left(f_{n}\right)_{1}^{\infty}$ is a sequence of continuous linear forms with $f_{n} \in$ $U_{n}^{0} \backslash\left(\operatorname{span} U_{n-1}^{0}\right)$, then $\left\{f_{n}: n=1,2, \ldots\right\}$ is linearly independent;

(c) if $G=\operatorname{span}\left\{f_{n}: n=1,2, \ldots\right\}$, then $G$ is $\sigma\left(E^{\prime}, E\right)$-closed.

Proof. (a) The fact that $E$ is metrisable but non-normable gives a decreasing base $\left\{U_{n}: n=0,1,2, \ldots\right\}$ such that, for each $n \geq 1, U_{n}^{0} \nsubseteq \lambda_{n} U_{n-1}^{0}$ for any $\lambda_{n}$. If for some $n, \operatorname{span} U_{n}^{0}=\operatorname{span} U_{n-1}^{0}=L$, then with unit ball $U_{n}^{0}$, $L$ is a Banach space. Similarly $L$ is a Banach space with unit ball $U_{n-1}^{0}$. But these norm topologies cannot coincide since $U_{n}^{0} \nsubseteq \lambda_{n} U_{n-1}^{0}$ for any $\lambda_{n}$; hence $U_{n}^{0} \nsubseteq \operatorname{span} U_{n-1}^{0}$.

(b) This is immediate from (a).

(c) It is easy to see that $G \cap U_{n}^{0} \subseteq \operatorname{span}\left\{f_{1}, \ldots, f_{n}\right\}$ for each $n \geq 1$. Hence $G \cap U_{n}^{0}=\operatorname{span}\left\{f_{1}, \ldots, f_{n}\right\} \cap U_{n}^{0}$, which is $\sigma\left(E^{\prime}, E\right)$-closed. Since $E$ is fully complete, being a Fréchet space, $G$ is $\sigma\left(E^{\prime}, E\right)$-closed.

THEOREM. Suppose that $E$ is the strict inductive limit of a strictly increasing sequence $\left(E_{m}\right)$ of Fréchet spaces, and that some $E_{m}$ is non-normable. Then $E$ has a properly separable quotient.

Proof. We may suppose that $E_{1}$ is non-normable; then so are all $E_{m}$. First, we construct a sequence $\left(f_{n}\right)$ of continuous linear forms on $E$.

By Lemma 2(a), applied to $E_{1}$, there is a base $\left\{U_{1 n}: n=0,1, \ldots\right\}$ of absolutely convex neighbourhoods of $o$ in $E$, such that $U_{1 n}^{0} \nsubseteq \operatorname{span} U_{1, n-1}^{0}$, for $n=1,2, \ldots$. Hence for each $n \geq 1$ there is some $f_{1 n}$ continuous on $E_{1}$, with $f_{1 n} \in U_{1 n}^{0} \backslash\left(\operatorname{span} U_{1, n-1}^{0}\right)$. Now apply Lemma 1 with $F=E_{1}, E=$ $E_{2}$ and $V_{n}=U_{1 n}$ for each $n$. Then there is a base $\left\{U_{2 n}: n=0,1, \ldots\right\}$ of absolutely convex neighbourhoods of $o$ in $E_{2}$ with $U_{2 n} \cap E_{1}=U_{1 n}$ for all $n$. By the Corollary to Lemma 1 , there is an extension $f_{2 n}$ of $f_{1 n}$ to $E_{2}$ with $f_{2 n} \in U_{2 n}^{0} \backslash\left(\operatorname{span} U_{2, n-1}^{0}\right)$ for all $n \geq 1$.

Continue up the $\left(E_{m}\right)$, by induction, to get, for each $m$, a base $\left\{U_{m n}\right.$ : $n=0,1, \ldots\}$ of absolutely convex neighbourhoods of $o$ in $E_{m}$, and a sequence $\left\{f_{m n}: n=1,2, \ldots\right\}$ of continuous linear forms on $E_{m}$ with $f_{m n} \in$ $U_{m n}^{0} \backslash\left(\operatorname{span} U_{m, n-1}^{0}\right)$. By Lemma 2 (b), $\left\{f_{m n}: n=1,2, \ldots\right\}$ is linearly independent.

On $E$, for each $n \geq 1$, define $f_{n}$ by $f_{n}(x)=f_{m n}(x)$ for all $x$ in $E_{m}$. Since $E=\bigcup_{m=1}^{\infty} E_{m}$ and $f_{m n}=f_{r n}$ on $E_{m}$ if $r \geq m, f_{n}$ is properly defined, and the 
restriction of $f_{n}$ to $E_{m}$ is $f_{m n}$. Thus $f_{n}$ is continuous on each $E_{m}$ and so on E.

Also $\left\{f_{n}: n=1,2, \ldots\right\}$ is linearly independent. For if $\sum_{1}^{k} \lambda_{n} f_{n}=o$ and no $f_{n}$ for $1 \leq n \leq k$ is $o$, then for each $n, 1 \leq n \leq k$, there is some $x_{n}$ with $f_{n}\left(x_{n}\right) \neq 0$ and $x_{n} \in E_{m_{n}}$, say. Put $m=\max \left\{m_{n}: 1 \leq n \leq k\right\}$. Then no $f_{n}$ is identically zero on $E_{m}$. But, restricting each $f_{n}$ to $E_{m}, \sum_{1}^{k} \lambda_{n} f_{m n}=o$. Since $\left\{f_{m n}: n=1,2, \ldots\right\}$ is linearly independent, $\lambda_{n}=0$ for $1 \leq n \leq k$.

Now let $G=\operatorname{span}\left\{f_{n}: n=1,2, \ldots\right\}$. Take $M=G^{0}$, the polar of $G$ in $E$, so that $M$ is a closed vector subspace of $E$. In the quotient topology on $E / M$, the dual is the polar $M^{0}$ of $M$ in $E^{\prime}$. Since $E$ is barrelled, the quotient topology is $\tau\left(E / M, M^{0}\right)$, or $\tau\left(E / G^{0}, G^{\infty}\right)$. We show next that $G^{00}=G$, that is, that $G$ is $\sigma\left(E^{\prime}, E\right)$-closed.

For each $m$, write $j_{m}$ for the injection of $E_{m}$ into $E$; then $j_{m}$ is continuous, $j_{m}^{\prime}$ is $\sigma\left(E^{\prime}, E\right)-\sigma\left(E_{m}^{\prime}, E_{m}\right)$ continuous and $j_{m}^{\prime}(f)$ is the restriction of $f$ to $E_{m}$. Thus $j_{m}^{\prime}\left(f_{n}\right)=f_{m n}$ for all $n \geq 1$, so $j_{m}^{\prime}(G)=G_{m}=\operatorname{span}\left\{f_{m n}: n=\right.$ $1,2, \ldots\}$. Hence $G \subseteq j_{m}^{\prime-1}\left(G_{m}\right)$, which is closed since $j_{m}^{\prime}$ is continuous and $G_{m}$ is $\sigma\left(E_{m}^{\prime}, E_{m}\right)$-closed, by Lemma $2(\mathrm{c})$. Therefore $G^{00}=\bar{G} \subseteq j_{m}^{\prime-1}\left(G_{m}\right)$ and so $j_{m}^{\prime}\left(G^{00}\right) \subseteq G_{m}$.

Now let $g \in G^{00}(g \neq o)$. There is some $m$ for which $g$ is not identically zero on $j_{m}\left(E_{m}\right)$; let $r$ be the least such $m$. Then $j_{r}^{\prime}(g) \in G_{r}$ and so

$$
j_{r}^{\prime}(g)=\sum_{n=1}^{k} \lambda_{n} f_{r n}=j_{r}^{\prime}\left(\sum_{n=1}^{k} \lambda_{n} f_{n}\right) \quad\left(\lambda_{k} \neq 0\right) .
$$

Now if $m>r, j_{m}^{\prime}(g) \in G_{m}$ and so

$$
j_{m}^{\prime}(g)=\sum_{n=1}^{p(m)} \lambda_{m n} f_{m n} \quad\left(\lambda_{m p(m)} \neq 0\right) .
$$

Hence, writing $j$ for the injection of $E_{r}$ into $E_{m}$, so that $j^{\prime}$ is the restriction of the elements of $E_{m}^{\prime}$ to $E_{r}$, we obtain

$$
\begin{gathered}
j_{r}=j_{m} \circ j \text { and } j_{r}^{\prime}=j^{\prime} \circ j_{m}^{\prime}, \\
j_{r}^{\prime}(g)=j^{\prime}\left(\sum_{n=1}^{p(m)} \lambda_{m n} f_{m n}\right)=\sum_{n=1}^{p(m)} \lambda_{m n} f_{r n}
\end{gathered}
$$

since $f_{m n}$ is an extension of $f_{r n}$ from $E_{r}$ to $E_{m}$. Thus

$$
\sum_{n=1}^{k} \lambda_{n} f_{r n}=\sum_{n=1}^{p(m)} \lambda_{m n} f_{r n}
$$


Now by Lemma 2(c), $\left\{f_{r n}: n=1,2, \ldots\right\}$ is linearly independent and so $p(m)=k$ and $\lambda_{m n}=\lambda_{n}$ for all $n$. Hence $j_{m}^{\prime}(g)=\sum_{1}^{k} \lambda_{n} f_{m n}=j_{m}^{\prime}\left(\sum_{1}^{k} \lambda_{n} f_{n}\right)$ and $g-\sum_{1}^{k} \lambda_{n} f_{n} \in j_{m}^{\prime-1}(o)$ for all $m \geq r$. Thus $g-\sum_{1}^{k} \lambda_{n} f_{n}$ is zero on each $j_{m}\left(E_{m}\right)$ for $m \geq r$, and so is zero on $E$ (and in fact $r=1$ ). Hence $g \in G$, and $G$ is closed.

Finally, we show that $E / G^{0}$ is properly separable. Since $G^{00}=G$, the quotient topology is $\tau\left(E / G^{0}, G\right)$. The dimension of $G$ is countable and so $\tau\left(E / G^{0}, G\right)$ is metrisable, and equal to $\sigma\left(E / G^{0}, G\right)$. Thus $E / G^{0}$ is a dense vector subspace of $G^{*}$ under $\sigma\left(G^{*}, G\right)$. But since $G$ has countable dimension, $G^{*} \cong \omega$ and so is separable and metrisable. Hence $E / G^{0}$ is metrisable, separable and also barrelled; thus $E / G^{0}$ is properly separable (as shown in the remarks following the definition of properly separable).

COROLLARY. With the hypotheses of the theorem, E has a metrisable properly separable quotient (from the proof).

EXAMPLE. It is shown in [3] that if there exists a Banach space $F$ with no separable quotient, then there also exists a strict inductive limit of Banach spaces with no properly separable quotient (namely $F \times \varphi$, isomorphic to the strict inductive limit of the spaces $F \times \varphi_{n}$, where $\varphi_{m}$ is $m$-dimensional).

\section{Added in Proof}

The following questions are still open:

(1) If $E$ is the strict inductive limit of a sequence of Banach spaces, each with a separable quotient, has $E$ a properly separable quotient?

(2) If $E$ is the strict inductive limit of a sequence of metrisable barrelled spaces, each with a separable quotient, has $E$ a properly separable quotient?

\section{References}

[1] G. Köthe, Topological vector spaces I (Springer-Verlag, 1969).

[2] H. E. Lacey, 'Separable quotients of Banach spaces', An. Acad. Brasil. Cienc. 44 (1972), 185-189.

[3] W. J. Robertson and P. P. Narayanaswami, On properly separable quotients and barrelled spaces, (Department of Mathematics, The University of Western Australia, Research Report, March 1988/1). 
[4] A. P. Robertson and W. J. Robertson, Topological vector spaces, 2nd ed. (Cambridge University Press, 1973).

[5] S. A. Saxon and A. Wilansky, 'The equivalence of some Banach space problems', Colloq. Math. 37 (1977), 217-226.

\section{Department of Mathematics}

University of Western Australia

Nedlands, W. A. 6009

Australia 